\title{
Assessment of wind turbine component loads under yaw-offset conditions
}

\author{
Rick Damiani, Scott Dana, Jennifer Annoni, Paul Fleming, Jason Roadman, Jeroen van Dam, and \\ Katherine Dykes \\ National Renewable Energy Laboratory, Golden, CO 80401, USA \\ Correspondence: Rick Damiani (rick.damiani@nrel.gov)
}

Received: 6 September 2017 - Discussion started: 12 September 2017

Revised: 27 November 2017 - Accepted: 19 December 2017 - Published: 13 April 2018

\begin{abstract}
Renewed interest in yaw control for wind turbine and power plants for wake redirection and load mitigation demands a clear understanding of the effects of running with skewed inflow. In this paper, we investigate the physics of yawed operations, building up the complexity from a simplified analytical treatment to more complex aeroelastic simulations. Results in terms of damage equivalent loads (DELs) and extreme loads under misaligned conditions of operation are compared to data collected from an instrumented, utility-scale wind turbine. The analysis shows that multiple factors are responsible for the DELs of the various components and that airfoil aerodynamics, elastic characteristics of the rotor, and turbulence intensities are the primary drivers. Both fatigue and extreme loads are observed to have relatively complex trends with yaw offsets, which can change depending on the wind-speed regime. Good agreement is found between predicted and measured trends for both fatigue and ultimate loads.
\end{abstract}

\section{Introduction}

Despite numerous studies on the skewed inflow and wake of wind turbines (e.g., Hand et al., 2001; Schepers, 2004, 2012; Schreck and Schepers, 2014; Fleming et al., 2015), uncertainties still remain on the loading effects experienced by their mechanical components under misaligned operation. Whereas new interest is directed at maximizing plant power performance via wake steering and yaw control (e.g., Gebraad et al., 2016), understanding and managing their effects on operation and maintenance (O\&M) costs are critical for the success of the strategy. For this reason, a clear understanding of the short- and long-term consequences of operating in skewed conditions, or with yaw offsets, is necessary to assess whether maintenance costs could change, and to facilitate intelligent ways to improve power performance that mitigate any negative O\&M impacts.

It can be argued that a certain degree of yaw error is always present during turbine operation, in part because of wind turbulence and in part because of mechanical errors in the turbine alignment and during yaw transients (cf. Fleming et al., 2014). Here we are concerned with the system- atic, skewed-inflow operation. The resulting wake redirection could significantly alleviate the cyclic loads of wind turbines located downwind of those operating off axis. Thomsen and Sørensen (1999) show that the increase in turbulence associated with operation in waked inflow can increase fatigue loads by up to $15 \%$ with respect to clean inflow. However, aerodynamic processes and loading effects on the upwind turbines are, to a degree, uncertain. A few studies have shown a reduction in blade load levels for turbines operating under certain yaw offsets, accompanied by an increase in loads for other components (e.g., drivetrain and tower; Kragh and Hansen, 2014; Zalkind and Pao, 2016). Some focus has also been given to the load variations under cyclic variations of angle of attack $(\alpha)$ and relative air velocity at the airfoil of interest $\left(U_{\text {rel }}\right)$, but conflicting information still exists. On the one hand, industry experts have either advised against yaw-offset operation (Huang, 2012; Bakhshi and Sandborn, 2016) or devised technology and algorithms to minimize yaw misalignment while maximizing power production at the individual turbines in a plant (Kragh and Hansen, 2015; Mortimer, 2016; Fleming et al., 2014). On the other hand, con- 
trol system scholars recommend adopting strategies that can redirect wakes away from downwind turbines to increase the plant power production as a whole (e.g., Dahlberg and Medici, 2003; Wagenaar et al., 2012; Jiménez et al., 2010; Gebraad et al., 2016), or even suggest the use of continuous yaw control to increase damping and reduce loads (e.g., Ekelund, 2000). In the middle, the owners and operators are left with the unsatisfying conundrum of increasing power production and associated revenues while taking the risk of accelerated machine damage and associated repair costs.

Data from wind tunnel experiments (e.g., Schepers et al., 2011; Schreck and Schepers, 2014) show load and performance trends under yawed conditions and emphasize the importance of dynamic stall and unsteady-aerodynamics (UA) effects; yet these tests do not capture the impact of realistic atmospheric turbulence on the loads of key wind-power-plant components. Some data from full-scale turbines are available (e.g., Boorsma, 2012), but they are generally associated with involuntary yaw errors rather than a systematic measurement campaign with prescribed yaw offsets and comparison to aeroelastic simulations.

In this paper, we examine the problem of skewed wake and inflow for one utility-scale turbine configuration. The analysis starts from a theoretical standpoint and builds up in complexity, going from a simplified, analytical model for the $\alpha$ and $U_{\text {rel }}$ azimuthal variations under different yaw offsets to a fully aeroelastic realization of the turbulent operation. At one end of the complexity spectrum, the analytical model is an aid to the designer, as it predicts load and performance outcomes under steady-state conditions and sheds light on the physics by highlighting the effects of shaft tilt, inflow shear, and yaw error. The aeroelastic model results, at the other end, account for turbulence effects and nonlinearities of the entire system and therefore provide more clarity about the systemdynamics response under realistic conditions. For practical predictions, the aeroelastic model should be validated; hence extensive data were collected on a $1.5 \mathrm{MW}$ turbine under several different yaw-offset conditions and compared to the model results.

In Sect. 2, we provide a description of the methods used in this study, the load channels of interest, and the turbine under analysis. In Sect. 3, the analytical model is presented. Section 4 provides results attained with simulations of the turbine operation under steady winds. Turbulent-inflow simulation results are compared to field-collected data in Sect. 5. A summary of the observations is offered in Sect. 6.

\section{Methods and load channels of interest}

Our analysis was focused on the General Electric (GE) 1.5sle 1.5 MW wind turbine (GEWT) (Mendoza et al., 2015) installed at the National Wind Technology Center (NWTC), which is owned by the U.S. Department of Energy (DOE) and operated by the National Renewable Energy Laboratory
(NREL). The turbine is instrumented with an extensive set of load and performance sensors per IEC (2015), which is described in the next section. An original GE aeroelastic model was modified to be used with NREL's aero-hydroservo-elastic tool for wind turbine design (FAST) version 8 (FASTv8) (Jonkman et al., 2015). FAST is a widely used industry and academic tool for load estimation.

One of the underlying goals of this paper was to link the observed results to the physics of the blade aerodynamics and structural dynamics. Therefore, we started with a simplified model of the aerodynamics under steady, but sheared, wind conditions and examined the azimuthal variation of the lift. An analytical model based on the variance of the lift out-ofplane component as a function of yaw offset was produced. The lift standard deviation, i.e., the square root of its variance and hence the associated blade load standard deviation, was used as an indicator of oscillating fatigue loads. We then examined how this model performed under different hubheight wind speeds and then increased complexity by first using AeroDyn 15 (AD15), FASTv8's aerodynamics module, in stand-alone mode and then using a fully aeroelastic model of the turbine within FASTv8. We demonstrated the effects of UA and of the structural response on the bladeroot bending moments by turning on and off FASTv8's UA module and the relevant structural degrees of freedom. This initial analysis was done based on two wind speeds, in turbine power regions 2 and 2.5. Finally, the model was further refined by accounting for turbulent inflow and was compared to data from the field for several wind speeds up to rated power wind speed. Twenty turbulent seeds were considered, using the IEC (2005) normal turbulence model (NTM), and the calculated loads were compared to those extracted from wind-speed binned data coming from the field. For the sake of brevity, results for only two wind speeds are shown, which, however, are representative of conditions at and below rated power.

Seven output channels of interest were selected: bladeroot edgewise bending moment (EBM) and flapwise bending moment (FBM) and their resultant root-bending moment (RBM); low-speed-shaft torque (LSSTq); tower-top bending moment (TTBM, resultant of the fore-aft and side-side components); tower-top torque (TTTq); and tower-base bending moment (TBBM, resultant of the fore-aft and side-side components). Initially, the focus was on the FBM because conflicting information exists in the literature and in the industry experience (Huang, 2012; Bakhshi and Sandborn, 2016), and because it is also associated with thrust loads responsible for structural stresses on downstream components. The analysis was mainly carried out on fatigue loading, and the results are given in terms of short-term damage equivalent loads (DELs) via rain flow counting (Downing and Socie, 1982). Note that we also provide some insights on ultimate loading, but they do not include parked and fault conditions. Simulations and field data are based on $10 \mathrm{~min}$ interval sets. In the next section, we describe the field instrumentation setup. 


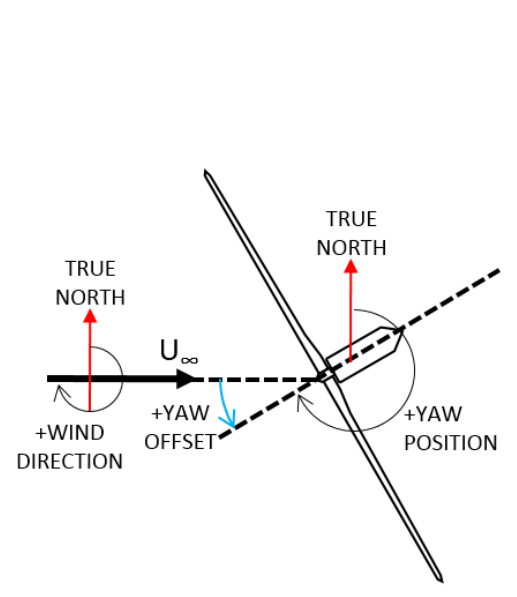

(a)

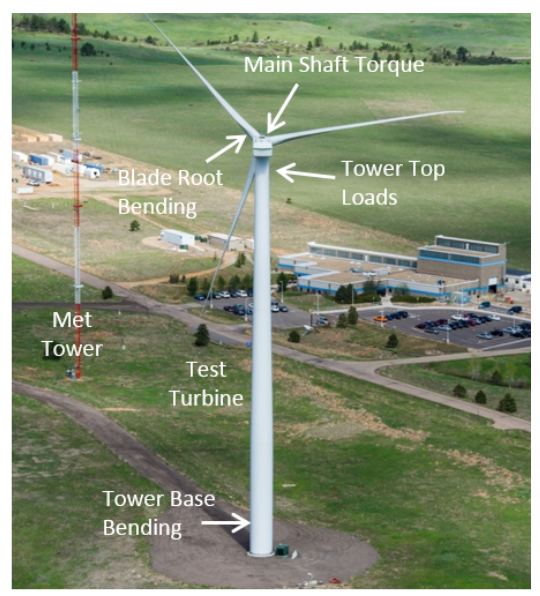

(b)

Figure 1. (a) Convention used in this paper for positive yaw and yaw offset. (b) View of the test turbine and meteorological tower, NREL 25911.

Table 1. Test turbine details.

\begin{tabular}{lr}
\hline $\begin{array}{l}\text { Brand and model } \\
\text { parameter }\end{array}$ & $\begin{array}{r}\text { GE 1.5sle ESS } \\
\text { value }\end{array}$ \\
\hline Rated power $(\mathrm{kW})$ & 1500 \\
Hub height $(\mathrm{m})$ & 80 \\
Nominal rotor diameter $(\mathrm{m})$ & 77 \\
Rated wind speed $\left(\mathrm{m} \mathrm{s}^{-1}\right)$ & 14 \\
\hline
\end{tabular}

\section{Field instrumentation}

The field-test campaign was carried out at the NWTC near Boulder, Colorado, and occurred over 6 months, during which the test turbine was operated with discrete yaw offsets over a range of wind speeds and atmospheric conditions.

The test turbine and its dedicated meteorological tower are shown in Fig. 1. Details of the turbine are provided in Table 1.

The turbine was instrumented in accordance with IEC 61400-13 (IEC, 2015) for mechanical-load measurements. All measurement methods and load processing were in conformance with this standard. Specifically, the tower base, tower top, and blade roots were instrumented for measurement of bending loads. In addition, the tower top and main shaft were instrumented for torque measurements. Encoders were used to measure yaw position, blade pitch, and rotor azimuth. The turbine was also instrumented for independent power measurements (per IEC, 2017). All signals were collected with a time-synchronous deterministic EtherCAT protocol and stored at a sample rate of $50 \mathrm{~Hz}$.

The meteorological tower is located $161 \mathrm{~m}(\sim 2$ rotor diameters) in the predominant upwind direction of the turbine and was instrumented in accordance with IEC 61400-12-1 (IEC, 2017). The meteorological tower measurement signals were time-synchronously recorded with the turbine loads sig- nals. For the analysis presented in this paper, the $80 \mathrm{~m}$ cup anemometer and the $87 \mathrm{~m}$ wind vane signal were used for the reference mean wind speed, direction, and turbulence intensity (TI). Test data were limited to a sector of directions in which the meteorological tower is largely upwind of the turbine. This measurement sector avoids any turbine wake influence on the meteorological tower instrumentation or influence of other turbines at the test site. More details on the instrumentation setup can be found in Dana et al. (2017).

The turbine nacelle's wind vane signal was modified using a frequency modulation device with a dedicated user interface for programming yaw-offset schedules of discrete offset values and durations. In no other way was the turbine or its controller manipulated. The turbine was set to cycle at $1 \mathrm{~h}$ intervals between the baseline setting, i.e., a $0^{\circ}$ yaw offset, and a nonzero offset. The offset values included $-25,-18$, $-12.5,0,12.5,18.0$, and $25.0^{\circ}$. However, not all offset value data could be used for analysis because of wind speeds or directions outside of the acceptance ranges selected for this study and discussed below.

As visible in Fig. 1, the convention employed for the sign of the yaw misalignment implies that with positive yaw offset the hub center is to the right of the tower centerline when looking downwind.

\section{Analytical simplification}

Fundamentally, the dominant loads during turbine operation originate from the aerodynamic forces and moments on the rotor blades. The largest contributor to the design loads on the blades and the downstream components is the lift distribution acting on the blade airfoils. Examining how the out-of-plane (rotor axial) component of the lift varies as a function of azimuth under different yaw angles can help explain the cyclic out-of-plane loading at the blade root and 


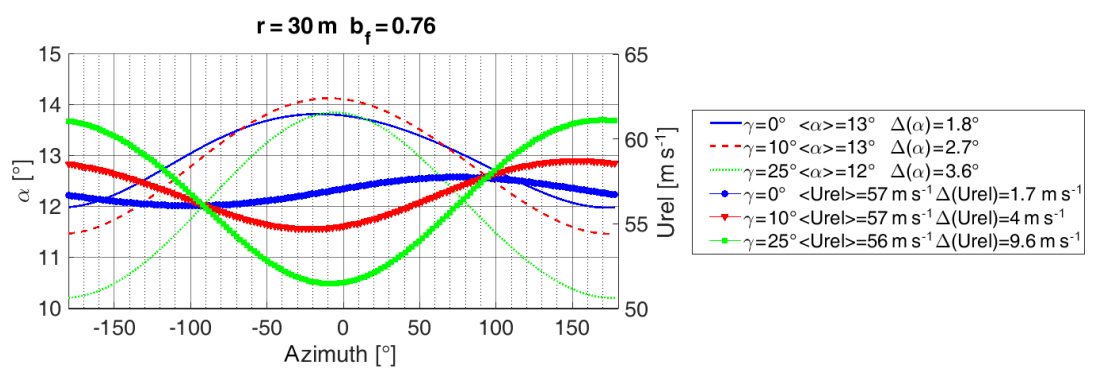

Figure 2. $\alpha$ (lines with no markers) and $U_{\text {rel }}$ (lines with markers) calculated by the simplified analytical model as a function of $\psi$ for the $75 \%$ blade-span station under yaw misalignments $(\gamma)$ as shown in the legend; $U_{\text {hub }}=12 \mathrm{~m} \mathrm{~s}^{-1}$. In the legend, the $<>$ and $\Delta$ denote mean and range (max-min) of the argument.

therefore the associated variation of several component loads (e.g., tower, drivetrain). The axial component $(\hat{l})$ of the 2-D airfoil lift can be written as

$\hat{l}=\frac{1}{2} \rho_{\mathrm{a}} U_{\mathrm{rel}}^{2} c_{1}(\alpha) c \cos \phi$,

where $\rho_{\mathrm{a}}$ is the air density, $c$ is the chord, $c_{1}(\alpha)$ is the 2-D lift coefficient, $\alpha$ is the angle of attack, $\phi$ is the inflow angle, and effects on $c_{1}$ due to Reynolds and Mach numbers are ignored. In an initial approximation, within the linear region of the airfoil lift polar, one could assume

$c_{1}(\alpha) \simeq c_{1} \alpha$,

where $c_{1}$ is an airfoil constant ( $\simeq 2 \pi$ for thin airfoils). As we are interested in fatigue loads, the standard deviation of $\hat{l}$ can be used as a surrogate for the load oscillation significance. Besides a constant, this translates to the standard deviation of the product between $c_{1} \cos \phi\left(\simeq c_{1} \alpha \cos \phi\right)$ and $U_{\text {rel }}^{2}$. To this end, through basic trigonometry, $\alpha$ and $U_{\text {rel }}$ can be written as in Eqs. (3)-(4):

$$
\begin{aligned}
& \alpha\left(\psi, r, \theta, U_{\mathrm{hub}}, \omega, \gamma\right)=\phi-\theta \\
& =\arctan \left(U\left(\psi, r, U_{\mathrm{hub}}\right) \cos (\gamma) \cos (\delta)\right) /\left(v_{t}(\omega, r)\right. \\
& \left.-U\left(\psi, r, U_{\mathrm{hub}}\right)[\cos (\gamma) \sin (\delta) \sin (\psi)+\sin (\gamma) \cos (\psi)]\right)-\theta,
\end{aligned}
$$

$U_{\text {rel }}\left(\psi, r, U_{\text {hub }}, \omega, \gamma\right)$

$=\sqrt{\left[U\left(\psi, r, U_{\mathrm{hub}}\right) \cos (\gamma) \cos (\delta)\right]^{2}+\left[v_{t}(\omega, r)-U\left(\psi, r, U_{\mathrm{hub}}\right)\right.}$

$\overline{[\cos (\gamma) \sin (\delta) \sin (\psi)+\sin (\gamma) \cos (\psi)]]^{2}}$,

where $\psi$ is the rotor azimuth angle, $r$ is the blade station radial position; $\theta$ is the airfoil aero-twist plus pitch setting; $\delta$ is the shaft tilt; $\omega$ is the rotor rotational speed; $\gamma$ is the rotor yaw offset; $v_{t}(\omega, r)$ is the airfoil tangential (in-plane) velocity; and $U\left(\psi, r, U_{\text {hub }}\right)$ is the wind speed at the elevation identified by $\psi$ and $r$ station, and a function of the $U_{\text {hub }}$, where $U_{\text {hub }}$ is the hub-height mean wind speed. Note that a power-law shear is included in $U\left(\psi, r, U_{\text {hub }}\right)$. Under the hypothesis of constant and uniform induction in region 2 of the turbine power-production curve, we ignore induction and tip effects that would translate into a constant bias in $\alpha$ and $c_{1}$ for most of the power-producing blade span.

In Fig. 2, $\alpha$ and $U_{\text {rel }}$ calculated by this analytical model are plotted as a function of $\psi$ for the $75 \%$ blade-span station under various yaw offsets and for $U_{\text {hub }}=12 \mathrm{~m} \mathrm{~s}^{-1}$ with a power-law shear exponent value of $0.2^{1}$. It can be seen that the impact of yaw offset reflects primarily on increasing the amplitude of the oscillations for both $\alpha$ and $U_{\text {rel }}$, with little change in the mean values. Of special note are the azimuthal loci of maxima and minima of the two quantities, which are shifted away from $\psi=0$ and $180^{\circ}$, demonstrating an asymmetry in the distribution of lift between the left and right halves of the rotor plane. The asymmetry is present even under aligned conditions, indicating that tilt and shear, beside yaw offset, are also contributing factors.

As shown by Eqs. (3)-(4) and Fig. 2, $\alpha$ and $U_{\text {rel }}$ are two quantities well correlated; thus we can use basic statistics (e.g., Frishman, 1975) to find the covariance of the product $c_{1} \cos \phi U_{\text {rel }}^{2}$ as in Eq. (5):

$$
\begin{aligned}
& \sigma^{2}\left(c_{1} \cos \phi \cdot U_{\text {rel }}^{2}\right) \simeq \sigma^{2}\left(\alpha \cos \phi \cdot U_{\text {rel }}^{2}\right)=\sigma_{\alpha \cos \phi \cdot U_{\text {rel }}^{2}}^{2} \\
& \quad=\sigma_{(\alpha \cos \phi)^{2}, U_{\text {rel }}^{4}}^{2}+\left[\sigma_{\alpha \cos \phi}^{2}+\overline{\alpha \cos \phi}^{2}\right] \cdot\left[\sigma_{U_{\text {rel }}^{2}}^{2}+{\overline{U_{\text {rel }}^{2}}}^{2}\right] \\
& -\left[\sigma_{\alpha \cos \phi, U_{\text {rel }}^{2}}^{2}+\overline{\alpha \cos \phi} \overline{U_{\text {rel }}^{2}}\right]^{2} .
\end{aligned}
$$

In Eq. (5), $\sigma^{2}$ denotes the covariance or variance of the argument, and overbars indicate the means of the variables; furthermore, the constant $c_{1}$ is omitted with no loss of generality.

In Fig. 3, the model-calculated standard deviations for the $75 \%$ span airfoil's $c_{1} \cos \phi, U_{\text {rel }}^{2}$, and their product are shown for different values of yaw offsets and hub-height wind speeds of 8 and $14 \mathrm{~m} \mathrm{~s}^{-1}$ with a wind shear exponent of 0.2 . The standard deviations were normalized by the means of their respective variables and are indicated as $\hat{\sigma}_{c_{1} \cos \phi}$,

\footnotetext{
${ }^{1}$ The sign convention adopted for the yaw offset is shown in Fig. 1.
} 
$\hat{\sigma}_{U_{\text {rel }}^{2}}$, and $\hat{\sigma}_{c_{1} \cos \phi \cdot U_{\text {rel }}^{2}}$, respectively. The rotor rotational speed was set at the average value obtained from aeroelastic simulations and verified against actual turbine field data for that wind speed.

The graphs show that, whereas $\hat{\sigma}_{U_{\text {rel }}^{2}}$ displays a minimum at $0^{\circ}$ yaw, the $\hat{\sigma}_{c_{1} \cos \phi}$ has a minimum near $-20^{\circ}$. By examining other wind-speed cases (not shown), which in general rendered similar trends to Fig. 3, it was observed that the minima in $\hat{\sigma}_{c_{1} \cos \phi}$ and $\hat{\sigma}_{c_{1} \cos \phi \cdot U_{\text {rel }}^{2}}$ get closer to the $\gamma=0^{\circ}$ location with increasing wind speed. Both the $\hat{\sigma}_{c_{1} \cos \phi}$ and $\hat{\sigma}_{U_{\text {rel }}^{2}}$ curves in Fig. 3 show an asymmetric behavior. The $\hat{\sigma}_{U_{\text {rel }}^{2}}$ standard deviation, for example, shows higher values for negative yaw offsets than for the corresponding positive misalignments. In contrast, $\hat{\sigma}_{c_{1} \cos \phi}$ exhibits larger variations for positive yaw offsets. The $\hat{\sigma}_{c_{1}} \cos \phi \cdot U_{\text {rel }}^{2}$ standard deviation, however, decreases sharply, going from negative to positive misalignments, with a reduction of some $66 \%$ at $\gamma=25^{\circ}$ with respect to the baseline case of $\gamma=0^{\circ}$. This simplified analysis suggests that, at least in terms of aerodynamic load oscillations, positive yaw misalignments could lead to a considerable load range reduction. Similar trends were observed at other span locations (not shown).

Although this analysis only considered steady winds, rigid blades, steady aerodynamics, and no induction, it brought forth results in agreement with recent studies. Zalkind and Pao (2016), for example, suggest the existence of an asymmetric behavior of blade and tower fatigue loads, hinting at the same positive offset as load-favorable misalignment direction.

\section{Blade aerodynamics and structural dynamics under steady winds}

To better assess the physics responsible for fatigue loading under skewed-inflow conditions, and to verify the existence of a preferred misalignment direction for loads, we build up models of increasing complexity utilizing AD15 and the full FASTv8 aeroelastic tool under steady winds.

Figure 4 shows azimuthal trends for $\alpha$ and $U_{\text {rel }}$ as calculated by AD15 for two GEWT blade-span stations (near 50 and $75 \%$ of the blade span) with aligned, sheared flow (0.2 power-law exponent) and no induction effects. It can be observed that $\alpha$ achieves a maximum before reaching the azimuthal zenith and a minimum affected by the tower shadow effects just before the $180^{\circ}$ azimuth; $U_{\text {rel }}$ exhibits an analogous trend, though shifted by $180^{\circ}$. The asymmetry is caused by the combination of the nonlinear wind profile (power law) and the trigonometric contribution of the airfoils' rotational speed to the relative air speed as a function of their positions (advancing/retreating effect). Therefore, the sole presence of shear and tilt causes an asymmetry in the variation of $c_{1}(\alpha)$ and $U_{\text {rel }}$ even under no-yaw-offset conditions, which can affect the performance of both the individual machine and the entire plant. For example, it can be speculated that under significant shear the optimum power performance could occur under misaligned conditions. Furthermore, the asymmetric distribution of $\alpha$ and $U_{\text {rel }}$ translates into an asymmetric azimuthal distribution of the induction factors, which in turn offsets the wake axis. A power increase under yaw-offset conditions is suggested by Kragh and Hansen (2014), and reports of skewed wake under aligned inflow conditions have been presented in Annoni et al. (2016) and Vollmer et al. (2016). These aspects are not further analyzed in this study, however.

By adding yaw offset, the asymmetry about the $0-180^{\circ}$ azimuth is still present (see also Fig. 2), and whereas the azimuthal mean $\alpha$ values slightly decrease with larger yaw offsets, the $\alpha$ ranges (max-min, denoted by " $\Delta$ ") increase with increasing offsets. Table 2 shows the range for $\alpha, c_{1}$, and $U_{\text {rel }}^{2}$ at different blade-span stations (given as spanwise fractions, $b_{\mathrm{f}}=0.28-0.92$ ) for two hub-height wind speeds ( 8 and $12 \mathrm{~m} \mathrm{~s}^{-1}$ ) and for $\gamma=0,-10$, and $10^{\circ}$. From the table, it can be observed that, relative to the aligned cases, $\alpha$ ranges reduce by $16-30 \%$ with negative misalignments and increase by $14-70 \%$ with positive ones. This is in agreement with the analytical model predictions. Also, when compared to the aligned cases, the $c_{1}$ ranges tend to be larger (smaller) for positive (negative) offsets. The $U_{\text {rel }}^{2}$ ranges, however, increase for both directions of misalignment but increase more under negative offsets. As these effects oppose each other when generating aerodynamic loads, it is difficult to ascertain whether one offset direction could lead to a more favorable load response. Furthermore, as shown in Table 2, the reduced frequencies $\left(k=\frac{\mathrm{d} \alpha}{\mathrm{d} t} c /\left(2 U_{\text {rel }}\right)\right.$, where $c$ is the chord length and $t$ denotes time) associated with the azimuthal $\alpha$ variations are largely above 0.02 , which is the commonly accepted threshold for unsteady aerodynamic effects to take place (Damiani et al., 2016). Given the expected higher values in angles of attack under positive yaw offsets, dynamic stall effects should be more prevalent with those misalignments.

Table 3 offers similar results to Table 2, but with induction, skewed wake correction (Ning et al., 2015), and UA effects included. For most blade spans and wind speeds, AD15 returned increased ranges of $\alpha$ with respect to the no-induction, geometric calculations. Overall, however, the trends explored under no-induction conditions still apply, with positive yaw offsets showing larger variations in $c_{1}$ but smaller in $U_{\text {rel }}^{2}$, confirming that the assumptions in the analytical model of constant and uniform induction are reasonable. By enabling UA (Damiani et al., 2016), higher peaks in $c_{1}$ 's especially inboard of the blade were noted. As visible in Fig. 5, which portrays azimuthal traces for $c_{1}$ at four blade-span stations with no UA (Fig. 5a) and with UA (Fig. 5b) effects, the impact of UA is mostly associated with a sharp stall transition for the inboard airfoils (at rotor radius $\leq 30 \%$ ), which is discussed below. 

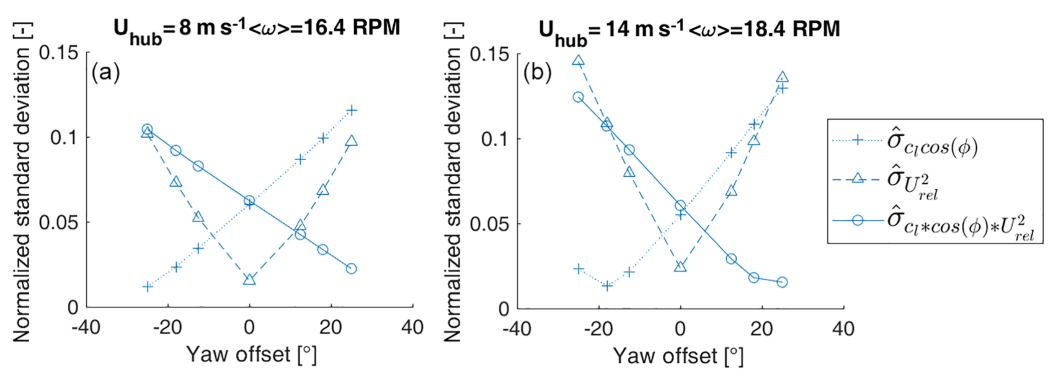

Figure 3. Standard deviations of $c_{1} \cos \phi$ (crosses), $U_{\text {rel }}^{2}$ (triangles), and their product (circles) for the airfoil at $\sim 75 \%$ of the blade span for two $U_{\text {hub }}$ wind speeds of $8 \mathrm{~m} \mathrm{~s}^{-1}$ (a) and $14 \mathrm{~m} \mathrm{~s}^{-1}$ (b), wind shear exponent value of 0.2 , and mean rotor rotational speed of $\omega=16.4$ (a) and $18 \mathrm{rpm}$ (b). The standard deviations are normalized by the means of the respective arguments.

$W S=8.0 \mathrm{~m} \mathrm{~s}^{-1} \mathrm{Yaw}=0^{\circ}$. nolnd.

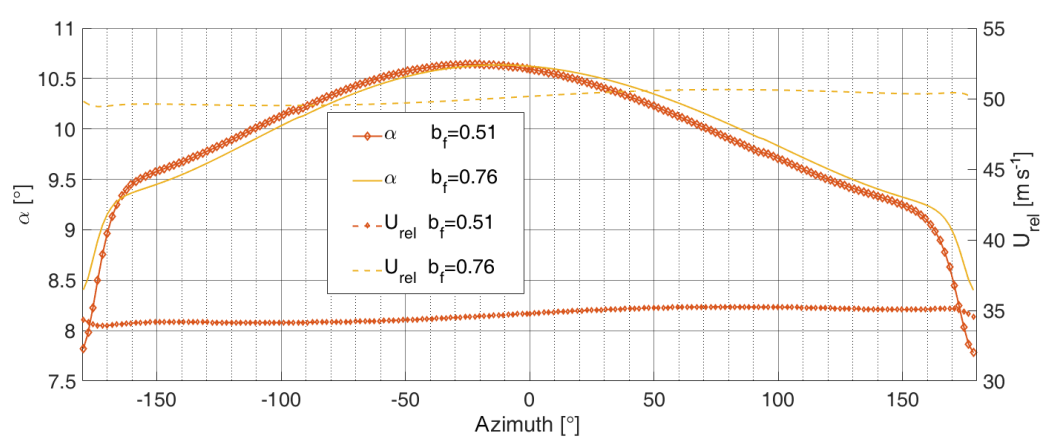

Figure 4. $\alpha$ and $U_{\text {rel }}$ for two blade stations (denoted in the legend by their spanwise fractions $b_{\mathrm{f}}$ ) as a function of $\psi$ under aligned conditions and a steady hub-height wind speed of $8 \mathrm{~m} \mathrm{~s}^{-1}$ as calculated by AD15.

Figure 6a shows the basic statistics of the blade-root FBM and EBM as calculated by a complete FASTv8 model of the turbine under investigation for yaw offsets ranging from -25 to $25^{\circ}$ and for 8 and $12 \mathrm{~m} \mathrm{~s}^{-1}$ hub-height wind speeds. The companion Fig. $6 \mathrm{~b}$ and c offer similar results, obtained by excluding either UA or the rotor/tower flexibility, respectively. In the plots, the 25th and 75th percentiles of the loads' distributions are marked with the left and right sides of rectangular boxes, respectively; the line in the middle of each box is the median, and the whisker lines show the distances to the most extreme sample values within the 1.5 interquartile range (i.e., 1.5 times the width of the box). Data beyond 1.5 times the interquartile ranges are shown as outliers with red crosses. Figure 6a shows little variation of the EBM statistics as a function of yaw offset and wind speed; this outcome is to be expected, as the EBM is mainly driven by gravity loads. The FBM median is seen to decrease with nonzero yaw offsets, as one would expect given that the mean thrust load reduces. The FBM distribution interquartile, and hence the variance and standard deviation, however, get larger with nonzero offsets, and more pronouncedly so for negative yaw offsets. Note that, in contrast to this result, the analytical model of Sect. 3 predicted an increase in variance for negative yaw offsets but a decrease for positive misalignments.
Data associated with Fig. 6 results demonstrate that the standard deviations tend to increase (except at the highest yaw offset) when UA is excluded from the computations. This indicates that UA acts as a low-pass filter on the spanintegral load. Furthermore, under rigid conditions, the FBM values are generally associated with slightly lower standard deviations, whereby it can be expected they would lead to lesser fatigue loads. These observations are confirmed by an analysis of the load variation as a function of azimuth (not shown for the sake of brevity), which translate into smoother curves.

In Fig. 7, the calculated short-term FBM DELs are presented for $U_{\text {hub }}=8$ and $12 \mathrm{~m} \mathrm{~s}^{-1}$, and for three different configurations: one including the entire model physics, one without UA effects, and one without blade (and tower) flexibility. The complete model results show a minimum of the DEL for the aligned conditions, although the asymmetry in the loading persists, with negative yaw offsets rendering larger loads than the positive counterparts. The second set of results in Fig. 7 is only slightly different from the first and thus confirms that the dynamic stall effects are not so important for the trends observed in blade-root loads, despite showing a noticeable effect on the $c_{1}$ 's as shown in Fig. 5. This result suggests that the integral of the lift distribution along the 


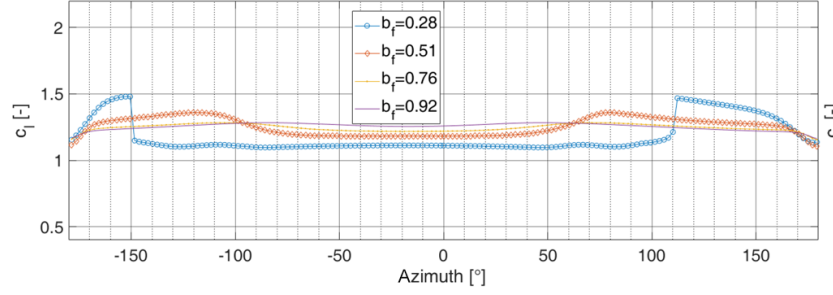

(a) Without UA

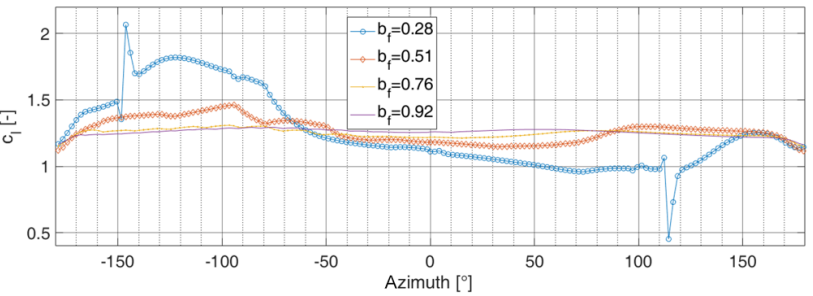

(b) With UA

Figure 5. (a) $c_{1}$ vs. $\psi$ for various blade stations $\left(b_{\mathrm{f}}\right)$ and for $\gamma=10^{\circ}$ offset as calculated by AD15 for a hub-height wind speed of $12 \mathrm{~m} \mathrm{~s}{ }^{-1}$. (b) As in (a) but with UA effects included.

Table 2. Ranges $(\Delta)$ for $\alpha, c_{1}$, and $U_{\text {rel }}^{2}$ as calculated by AD15 (without induction) for various blade stations $\left(b_{\mathrm{f}}\right)$ and hub-height wind speeds $\left(U_{\text {hub }}\right)$ and for yaw offsets $(\gamma)$ of $0,-10$, and $10^{\circ}$. A wind shear exponent of 0.2 was assumed. The calculated reduced frequencies $(k)$ for the various airfoil stations are also given.

\begin{tabular}{|c|c|c|c|c|c|c|c|c|c|c|c|}
\hline \multirow{2}{*}{$\begin{array}{l}U_{\text {hub }} \\
{\left[\mathrm{m} \mathrm{s}^{-1}\right]}\end{array}$} & \multirow{2}{*}{$\begin{array}{l}b_{\mathrm{f}} \\
{[-]}\end{array}$} & \multicolumn{4}{|c|}{$\gamma=0^{\circ}$} & \multicolumn{3}{|c|}{$\gamma=-10^{\circ \mathrm{a}}$} & \multicolumn{3}{|c|}{$\gamma=10^{\circ \mathrm{a}}$} \\
\hline & & $\begin{array}{r}\Delta(\alpha) \\
{\left[{ }^{\circ}\right]}\end{array}$ & $\begin{array}{r}k \\
{[-]}\end{array}$ & $\begin{array}{r}\Delta\left(c_{1}\right) \\
{[-]}\end{array}$ & $\begin{array}{c}\Delta\left(U_{\mathrm{rel}}^{2}\right) \\
{\left[\mathrm{m}^{2} \mathrm{~s}^{-2}\right]}\end{array}$ & $\begin{array}{r}\Delta^{*}(\alpha) \\
{[-]}\end{array}$ & $\begin{array}{r}\Delta^{*}\left(c_{1}\right) \\
{[-]}\end{array}$ & $\begin{array}{r}\Delta^{*}\left(U_{\mathrm{rel}}^{2}\right) \\
{[-]}\end{array}$ & $\begin{array}{r}\Delta^{*}(\alpha) \\
{[-]}\end{array}$ & $\begin{array}{r}\Delta^{*}\left(c_{1}\right) \\
{[-]}\end{array}$ & $\begin{array}{r}\Delta^{*}\left(U_{\mathrm{rel}}^{2}\right) \\
{[-]}\end{array}$ \\
\hline \multirow[t]{4}{*}{8} & 0.28 & 4.4 & 0.11 & 0.38 & 63 & -0.16 & -0.05 & 1.47 & 0.57 & 0.00 & 1.07 \\
\hline & 0.51 & 2.9 & 0.046 & 0.11 & 91 & -0.31 & -0.19 & 1.85 & 0.31 & 0.45 & 1.46 \\
\hline & 0.76 & 2.2 & 0.02 & 0.09 & 120 & -0.18 & -0.15 & 1.92 & 0.23 & 0.24 & 1.58 \\
\hline & 0.92 & 2.1 & 0.011 & 0.09 & 132 & -0.19 & -0.14 & 2.18 & 0.14 & 0.17 & 1.73 \\
\hline \multirow[t]{4}{*}{12} & 0.28 & 5.7 & 0.11 & 0.03 & 115 & -0.14 & 0.07 & 1.39 & 0.70 & 0.30 & 0.87 \\
\hline & 0.51 & 3.7 & 0.045 & 0.06 & 168 & -0.32 & -0.63 & 1.62 & 0.46 & 1.68 & 1.14 \\
\hline & 0.76 & 3 & 0.02 & 0.07 & 217 & -0.30 & -0.10 & 1.84 & 0.27 & 0.01 & 1.37 \\
\hline & 0.92 & 2.7 & 0.011 & 0.07 & 245 & -0.22 & -0.01 & 1.94 & 0.22 & 0.01 & 1.44 \\
\hline
\end{tabular}

a $\Delta^{*}$ denotes the relative difference in range of the argument, with respect to the same quantity at $0^{\circ}$ yaw offset.

blade span washes out the UA contribution to the oscillating loads. Finally, with rigid blades and $U_{\text {hub }}=8 \mathrm{~m} \mathrm{~s}^{-1}$, the trend for the root FBM DEL is as expected from the simplified variance model of Sect. 3. Therefore, at this wind speed, the inertial forces associated with the elastic response - of the rotor in particular - have a dominant effect on the calculated DELs.

At $U_{\text {hub }}=12 \mathrm{~m} \mathrm{~s}^{-1}$, however, the DEL trend with yaw offset reflects what is observed in the first set of Fig. 7, suggesting that the load variation with yaw offset at this wind speed is only slightly affected by the structural response and therefore primarily driven by pure aerodynamic loading ${ }^{2}$. To understand what is happening at $12 \mathrm{~m} \mathrm{~s}^{-1}$, we further examined the blade $c_{1}$-vs.-azimuth relationship. Figure 8 shows how blade structural flexibility affects the $c_{1}$-vs.-azimuth trends for various blade-span stations at $\gamma=12.5^{\circ}$ and for $U_{\text {hub }}=8$ and $12 \mathrm{~m} \mathrm{~s}^{-1}$. At the higher wind speeds, the inboard airfoils start stalling, even at $\gamma=0^{\circ}$, and the abrupt drop in $c_{1}$ creates a load forcing that contributes to the higher DELs. This

\footnotetext{
${ }^{2}$ Note that the turbine controller constantly varies the pitch of the blades at this speed and at all the yaw errors examined thus far, but it was verified that disabling the controller in the simulations does not significantly affect these observations.
}

can also be observed in Fig. 5. By comparing Fig. 8a to b, one can note the $100 \%$ increase in the $c_{1}$ range for the station near the $30 \%$ blade span when going from $U_{\text {hub }}=8 \mathrm{~m} \mathrm{~s}^{-1}$ to $U_{\text {hub }}=12 \mathrm{~m} \mathrm{~s}^{-1}$. Furthermore, by comparing Fig. $8 \mathrm{a}-\mathrm{b}$ to Fig. $8 \mathrm{c}-\mathrm{d}$, one can see that, at $U_{\text {hub }}=12 \mathrm{~m} \mathrm{~s}^{-1}$, the lift curves are almost unchanged when the blades are made rigid, as opposed to the case with $8 \mathrm{~m} \mathrm{~s}^{-1}$ hub-height wind speed, where the blade flexibility returns a more complex $c_{1}$ response in the region of tower influence near the $180^{\circ}$ azimuth for the outboard airfoils, thus again leading to expected higher fatigue loading. This analysis explains why, at the higher wind speeds, the impact of flexibility is almost negligible on the FBM short-term DEL trend with yaw misalignment but how suppressing elastic response at the lower wind speeds can significantly change the fatigue loading level.

Figure 9 further demonstrates how the blade inboard stations exhibit a sharp drop in lift near stall. The combination of higher $\alpha$ 's with higher wind speeds, and the sharper airfoil stall characteristics of the inboard airfoils, causes the large variations in $c_{1}$ 's, which in turn are responsible for the increase in oscillating loads at the root.

This nonlinear aerodynamics explains why the simple variance model cannot capture load trends at higher wind 
Table 3. Ranges $(\Delta)$ for $\alpha, c_{1}$, and $U_{\text {rel }}^{2}$ as calculated by AD15 (with induction) for various blade stations $\left(b_{\mathrm{f}}\right)$ and hub-height wind speeds $\left(U_{\text {hub }}\right)$ and for yaw offsets $(\gamma)$ of $0,-10$, and $10^{\circ}$. A wind shear exponent of 0.2 was assumed. Results for $\Delta\left(c_{1}\right)$ are given with (UA) and without (nUA) unsteady aerodynamics effects included.

\begin{tabular}{|c|c|c|c|c|c|c|c|c|c|c|c|c|c|}
\hline \multirow{3}{*}{$\begin{array}{l}U_{\text {hub }} \\
{\left[\mathrm{m} \mathrm{s}^{-1}\right]}\end{array}$} & \multirow{3}{*}{$\begin{array}{l}b_{\mathrm{f}} \\
{[-]}\end{array}$} & \multicolumn{4}{|c|}{$\gamma=0^{\circ}$} & \multicolumn{4}{|c|}{$\gamma=-10^{\circ \mathrm{a}}$} & \multicolumn{4}{|c|}{$\gamma=10^{\circ \mathrm{a}}$} \\
\hline & & \multirow{2}{*}{$\begin{array}{r}\Delta(\alpha) \\
{\left[{ }^{\circ}\right]}\end{array}$} & \multicolumn{2}{|c|}{$\Delta\left(c_{1}\right)$} & \multirow{2}{*}{$\begin{array}{c}\Delta\left(U_{\text {rel }}^{2}\right) \\
{\left[\mathrm{m}^{2} \mathrm{~s}^{-2}\right]}\end{array}$} & \multirow{2}{*}{$\begin{array}{r}\Delta^{*}(\alpha) \\
{[-]}\end{array}$} & \multicolumn{2}{|c|}{$\Delta^{*}\left(c_{1}\right)$} & \multirow{2}{*}{$\begin{array}{r}*\left(U_{\mathrm{rel}}^{2}\right) \\
{[-]}\end{array}$} & \multirow{2}{*}{$\begin{array}{r}\Delta^{*}(\alpha) \\
{[-]}\end{array}$} & \multicolumn{2}{|c|}{$\Delta^{*}\left(c_{1}\right)$} & \multirow{2}{*}{$\begin{array}{r}\Delta^{*}\left(U_{\mathrm{rel}}^{2}\right) \\
{[-]}\end{array}$} \\
\hline & & & nUA [-] & UA [-] & & & nUA [-] & UA [-] & & & nUA [-] & UA [-] & \\
\hline \multirow[t]{4}{*}{8} & 0.28 & 3.2 & 0.37 & 0.31 & 63 & -0.16 & -0.16 & -0.26 & 1.47 & 0.63 & 0.59 & 0.65 & 1.07 \\
\hline & 0.51 & 2.6 & 0.3 & 0.27 & 91 & -0.31 & -0.30 & -0.37 & 1.69 & 0.38 & 0.40 & 0.44 & 1.38 \\
\hline & 0.76 & 2.6 & 0.26 & 0.24 & 120 & -0.19 & -0.35 & -0.21 & 1.92 & 0.27 & 0.23 & 0.25 & 1.58 \\
\hline & 0.92 & 2.1 & 0.2 & 0.19 & 132 & -0.14 & -1.00 & -0.11 & 2.18 & 0.24 & 0.20 & 0.26 & 1.73 \\
\hline \multirow[t]{4}{*}{12} & 0.28 & 8.9 & 0.38 & 1.7 & 115 & -0.07 & 0.00 & 0.00 & 1.39 & 0.46 & 0.00 & 0.12 & 0.87 \\
\hline & 0.51 & 4.9 & 0.17 & 0.25 & 168 & -0.37 & -0.35 & 0.04 & 1.62 & 0.39 & 0.47 & 0.40 & 1.14 \\
\hline & 0.76 & 3.5 & 0.089 & 0.12 & 217 & -0.34 & -0.16 & 0.00 & 1.84 & 0.29 & 0.46 & 0.33 & 1.37 \\
\hline & 0.92 & 2.9 & 0.093 & 0.1 & 245 & -0.24 & -0.09 & -0.10 & 1.94 & 0.24 & 0.29 & 0.30 & 1.44 \\
\hline
\end{tabular}

${ }^{\mathrm{a}} \Delta^{*}$ denotes the relative difference in range of the argument, with respect to the same quantity at $0^{\circ}$ yaw offset.

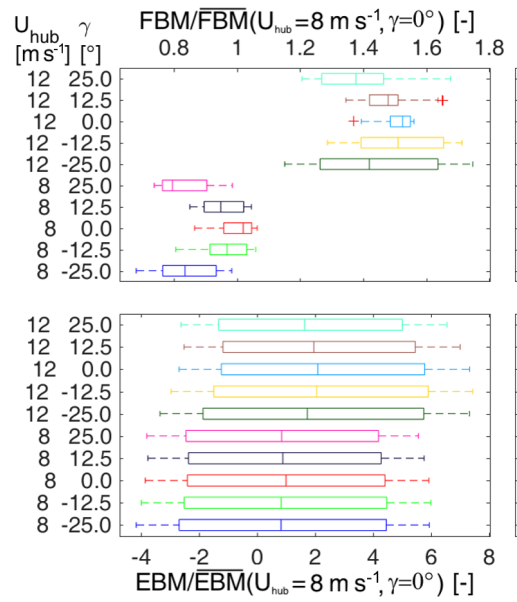

(a) Complete mode1
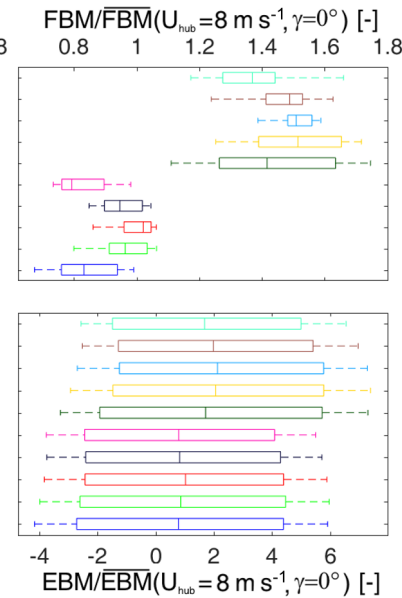

(b) No UA
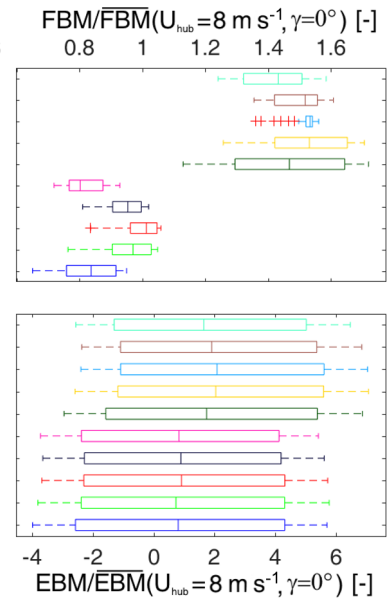

(c) Rigid blades

Figure 6. (a) Nondimensional FBM (top) and EBM (bottom) boxplot statistics for the various cases of $U_{\text {hub }}\left(8\right.$ and $\left.12 \mathrm{~m} \mathrm{~s}^{-1}\right)$ and $\gamma(-25$ to $25^{\circ}$ ) as indicated along the $y$ axis, as calculated by FASTv8 for a complete model of the GEWT. The loads are normalized by the respective means at $8 \mathrm{~m} \mathrm{~s}^{-1}$ and $0^{\circ}$ yaw offset. (b) As in (a) but calculated excluding UA effects, and (c) as in (a) but calculated with rigid blades and tower.

speeds and therefore indicates that the airfoil characteristics and the associated UA, together with the elastic response of the blades, contribute to the actual fatigue loading of the blade and thus all the downstream turbine components. Given these results, one can only rely on accurate, nonlinear, aeroelastic models of the turbine and thorough loads analyses to assess the loading levels in the various components when operating under yaw offsets. In the subsequent section, we carry out a load analysis based on FASTv8 simulations with turbulent winds and compare the predictions to fieldmeasured loads.

\section{Aeroelastic simulation and field data validation}

To compare model predictions to actual field measurements, we ran aeroelastic simulations for hub-height mean wind speeds ranging from 4 to $24 \mathrm{~m} \mathrm{~s}^{-1}$ in $2 \mathrm{~m} \mathrm{~s}^{-1}$ wind-speed bins, and with NTM inflow (see also IEC, 2005) with 20 turbulence seeds per wind-speed bin. However, besides brevity, we limit the discussion to mean hub-height wind speeds of 10 and $14 \mathrm{~m} \mathrm{~s}^{-1}$ for three reasons. First, we intend to compare to data bins from the field; thus we selected those that provided sufficient field data points for statistical significance. Second, above rated wind speed, pitch dynamics add more complexity to the interpretation of the results. Lastly, low wind speeds $\left(\leq 6 \mathrm{~m} \mathrm{~s}^{-1}\right)$ gave rise to shutdowns in the simulations, which we wanted to exclude for the sake of clarity. Furthermore, the selected wind speeds are representative of conditions at or below rated power, which is where wake steering is thought to be most effective (e.g., Gebraad et al., 2017).

The investigated yaw offsets ranged from -25 to $+25^{\circ}$, which have been considered for wake redirection in wind 


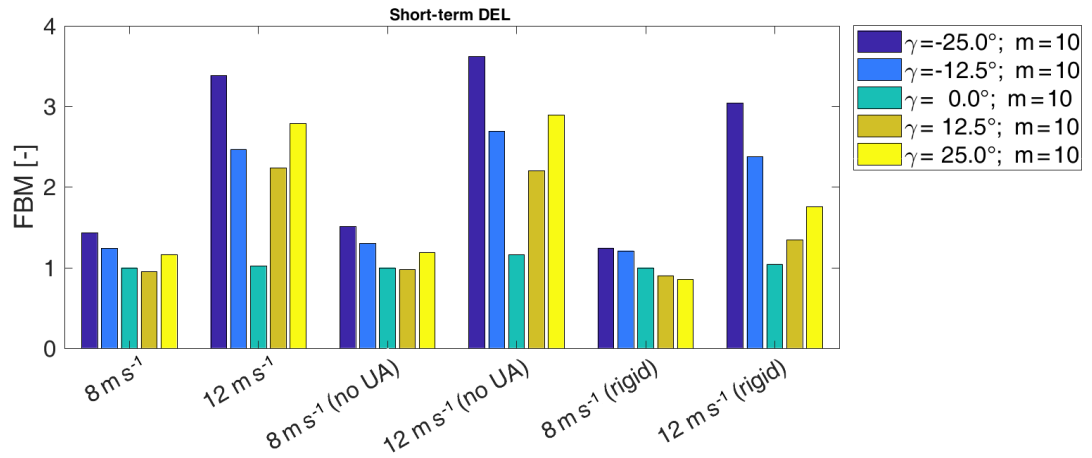

Figure 7. Blade-root FBM short-term DEL with yaw offsets from -25 to $25^{\circ}$ as calculated by FASTv 8 for $U_{\text {hub }}=8$ and $12 \mathrm{~m} \mathrm{~s}{ }^{-1}$. Also shown are results obtained either by excluding UA (denoted by "no UA") or by removing structural rotor flexibility (denoted as "rigid").

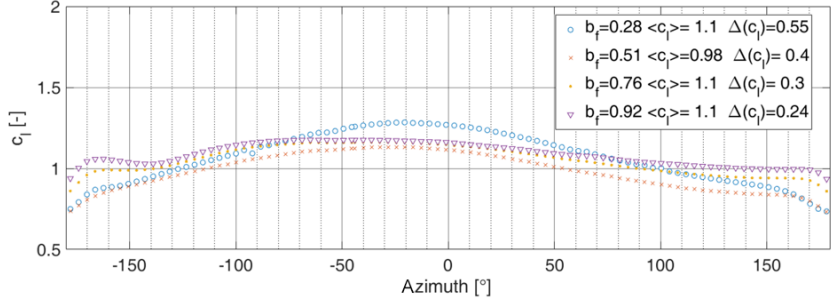

(a) $8 \mathrm{~m} \mathrm{~s}^{-1}$

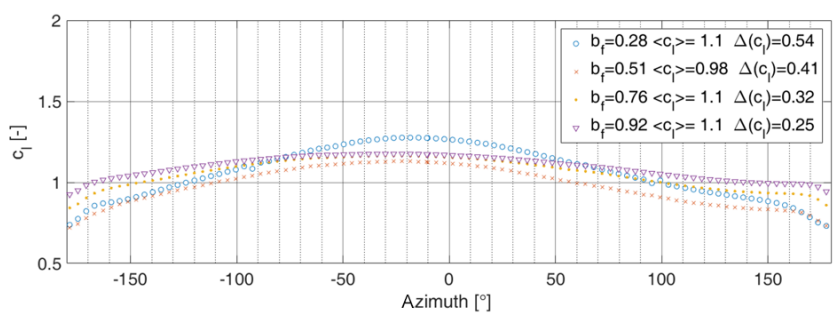

(c) $8 \mathrm{~m} \mathrm{~s}^{-1}$, rigid blades

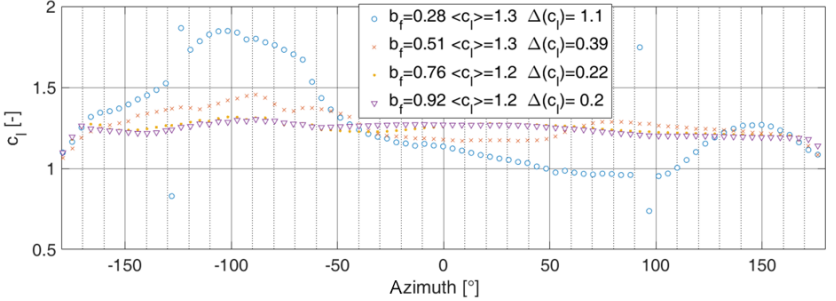

(b) $12 \mathrm{~m} \mathrm{~s}^{-1}$

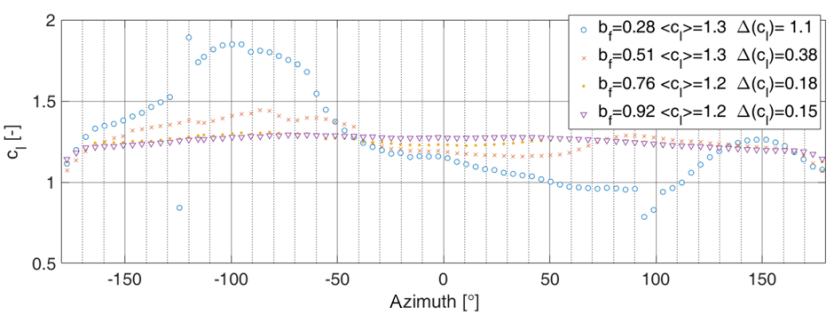

(c) $12 \mathrm{~m} \mathrm{~s}^{-1}$, rigid blades

Figure 8. FASTv8 results with steady winds; (a) $c_{1}$ vs. $\psi$ for $8 \mathrm{~m} \mathrm{~s}^{-1}$ wind speed and $12.5^{\circ}$ yaw offset, (b) as in (a) for a hub-height wind speed of $12 \mathrm{~m} \mathrm{~s}^{-1}$, and $(\mathbf{c}, \mathbf{d})$ as in $(\mathbf{a}, \mathbf{b})$ but with rigid blades and tower. Note that the legends provide mean $($ denoted by $<>)$ and range (max-min) of $c_{1}$ for the various span stations $\left(b_{\mathrm{f}}\right)$.

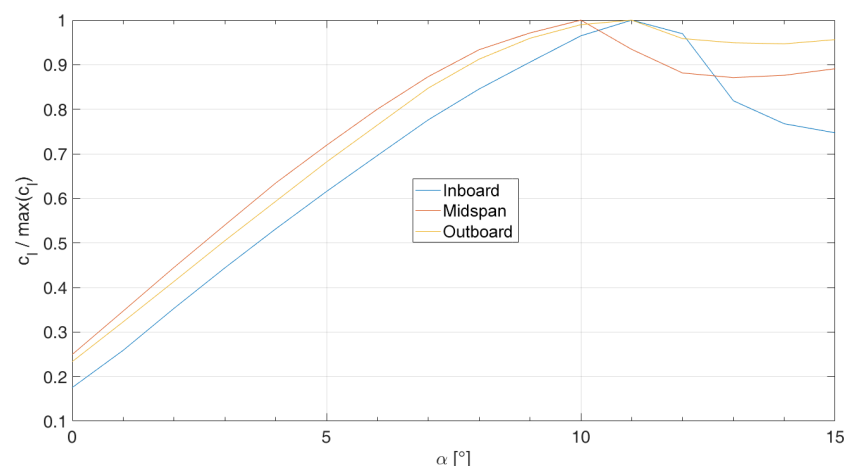

Figure 9. Lift polar curve of the inboard to midspan airfoils normalized to the respective maximum $c_{1}$ 's. farms. Because the field data showed large variations in TI within individual wind-speed bins (see Fig. 10), a lower turbulence range was selected for the simulations, with a mean $\mathrm{TI}=15 \%$ throughout the wind-speed range. Accordingly, the field data were filtered to retain measurements with TI ranging from 1 to $17 \%$ to guarantee a statistically significant number of samples. This was necessary because turbulence intensity proved to have a larger effect than the sole yaw offset on the magnitude of DELs, as can be seen, for example, in the blade-root FBM DELs shown in Fig. 11.

\subsection{Fatigue loads}

Figure 12 offers a summary comparison between predicted and measured short-term DELs for the various channels of interest and for 10 and $14 \mathrm{~m} \mathrm{~s}^{-1}$. The bars represent mean values from simulation results, and the square symbols are 

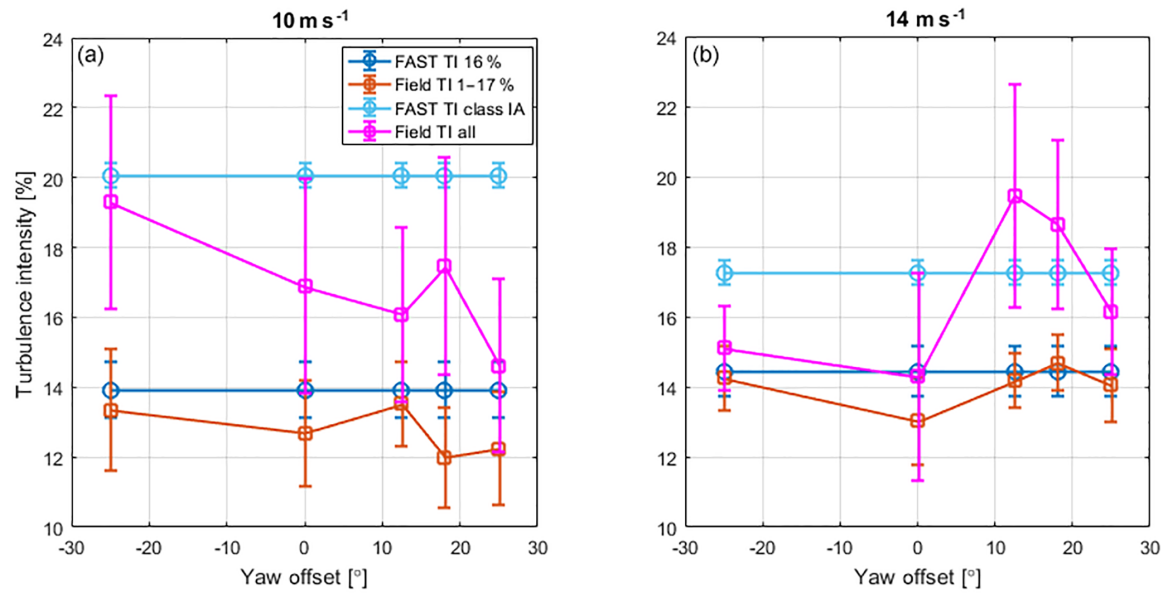

Figure 10. Comparison between simulated (circles) and measured (squares) values of TI as a function of yaw offset for two wind-speed bins of interest (a: $U_{\text {hub }}=10 \mathrm{~m} \mathrm{~s}^{-1} ; \mathbf{b}: U_{\text {hub }}=14 \mathrm{~m} \mathrm{~s}^{-1}$ ). The field data are shown when including all data (label "All" in the legend) and when filtering for TI below $17 \%$ (label "TI 1-17\%" in the legend). The simulated data are shown for class IA turbulence (IEC, 2005) (label "FAST TI Class IA" in the legend) and for TI $=16 \%$ at $15 \mathrm{~m} \mathrm{~s}^{-1}$ (label "FAST TI $\left.16 \% "\right)$.
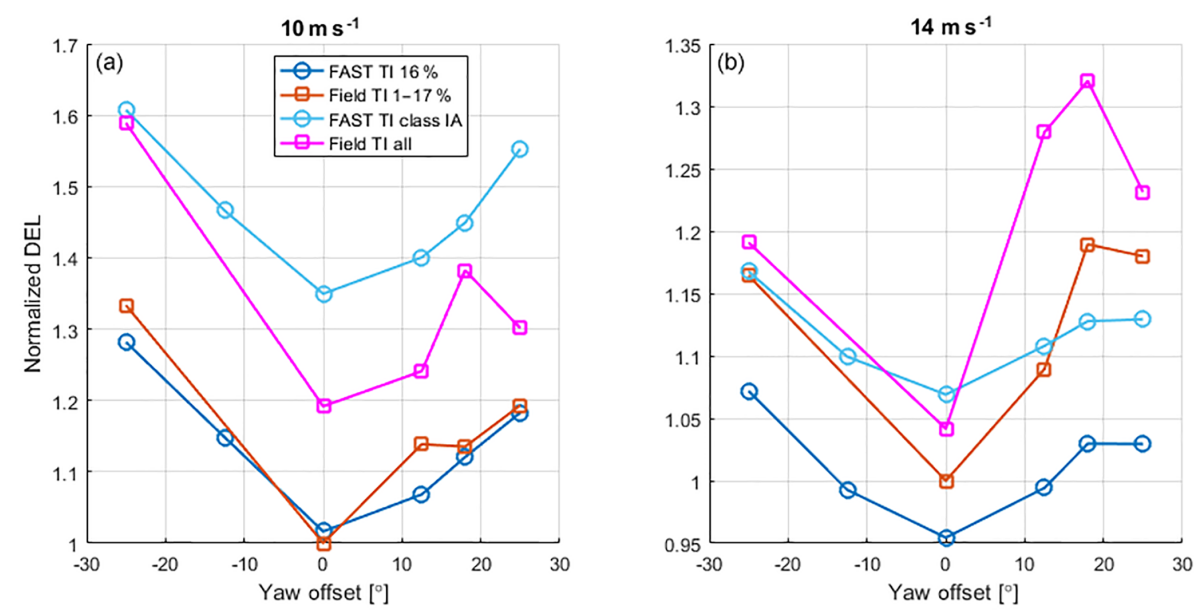

Figure 11. TI effect on the blade FBM DEL. Comparison between simulated (circles) and measured (squares) values of the loads for various TI levels, as a function of yaw offset and for $U_{\text {hub }}=10 \mathrm{~m} \mathrm{~s}^{-1}$ (a) and $U_{\text {hub }}=14 \mathrm{~m} \mathrm{~s}^{-1}$ (b). The loads are normalized by the mean value at $0^{\circ}$ yaw offset from the field data filtered to a TI range of 1 to $17 \%$. Labels in the legend are the same as in Fig. 10.

the corresponding values derived from the field measurements. For each channel, the mean values are given as a function of yaw offset, with all DELs normalized by the field value at the zero yaw offset. Because field data at $\gamma=-12.5^{\circ}$ were scarce, they were not included in the plots. In Appendix A, Figs. A1-A7 show detailed comparisons between the predicted and the measured DELs for the various load channels of interest as a function of yaw offset and including error bars representing \pm 1 standard deviations. The spread comes from multiple seeds in the FAST results and from multiple data points $(10 \mathrm{~min}$ records) in the field datasets.

Overall, good agreement was observed between predicted and measured data both in absolute terms and as far as yaw- offset trends are concerned. For example, the FBM values predicted at the blade root are very close to the corresponding field-derived data. A minimum is seen under aligned conditions as was also shown under steady-state wind conditions in Sect. 4. The previously observed asymmetry in the mean loading as a function of yaw offset is still visible, with negative yaw errors giving rise to larger loads, though at $14 \mathrm{~m} \mathrm{~s}^{-1}$ this effect is not confirmed by the field-derived data.

Both the calculated and measured blade-root EBM display a mostly monotonic, decreasing trend with increasing yaw offset, although less pronounced at $14 \mathrm{~m} \mathrm{~s}^{-1}$. This decreasing trend is in line with the decrease in standard deviations observed in Fig. 6a under steady conditions and is attributable 

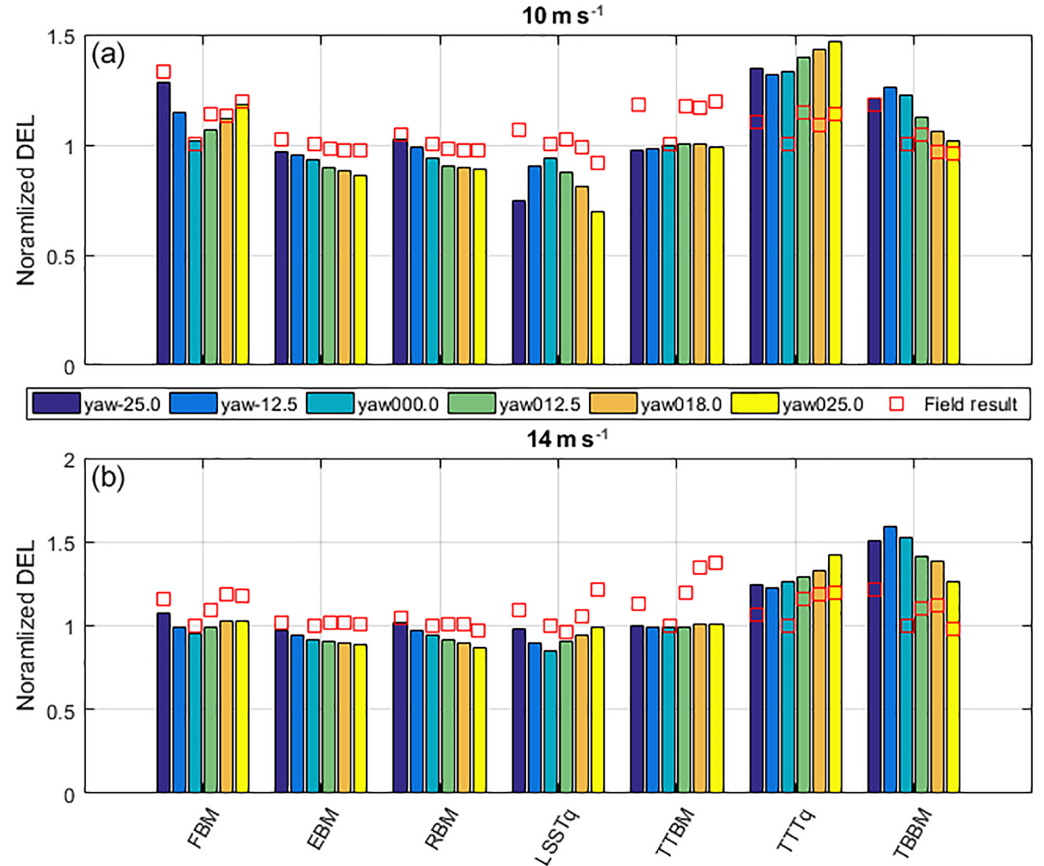

Figure 12. Comparison between predicted (FAST, bars) and measured (field, square symbols) mean DELs as a function of yaw offsets for mean $U_{\text {hub }}=10 \mathrm{~m} \mathrm{~s}^{-1}$ (a) and $14 \mathrm{~m} \mathrm{~s}^{-1}$ (b). DELs are normalized by their respective mean values at $0^{\circ}$ yaw offset as calculated from the field measurements for the respective wind-speed bins.

to the aerodynamics partially relieving the gravity loading when going from negative to positive yaw offsets.

To assess the effective fatigue load on the blade root, the resultant unsymmetric RBM must be analyzed via load roses, wherein multiple circular sectors of the root cross section are considered, in addition to the $0^{\circ}(\mathrm{FBM})$ and $90^{\circ}$ (EBM) components. Eight sectors, each $22.5^{\circ}$ wide, were analyzed, and the data from the largest contributing sector from each yaw offset were selected and normalized with respect to the field value under aligned conditions. The calculated results demonstrate that the resulting fatigue loading decreases with increasing yaw offset and that negative misalignments appear more detrimental than the aligned conditions. The fieldderived results generally confirm this trend, but not as clearly.

The LSSTq DELs also showed good agreement between predicted and measured data, especially at $14 \mathrm{~m} \mathrm{~s}^{-1}$. Note, however, the reversal in the trend with yaw misalignments for different wind speeds. For wind speeds below $14 \mathrm{~m} \mathrm{~s}^{-1}$ and near $\gamma=0^{\circ}$, in fact, FAST results exhibited a maximum in torque DEL, whereas they showed a minimum for a wind speed of $14 \mathrm{~m} \mathrm{~s}^{-1}$. The field-derived data confirm this reversal, although an outlier can be noted at $\gamma=-25^{\circ}$ and $10 \mathrm{~m} \mathrm{~s}^{-1}$.

A more significant discrepancy was observed for the mean resultant TTBM DEL. The load-rose analysis of field data (conducted in the same fashion as for the blade-root loads above) exhibits an increase in the short-term fatigue damage for nonzero yaw offsets. FAST predictions, however, render a much smaller effect of the yaw misalignment. The largest discrepancy between field and FAST results $(\sim 40 \%$ relative error) can be seen at $\gamma=25^{\circ}$ and $14 \mathrm{~m} \mathrm{~s}^{-1}$, but it is also accompanied by very large standard deviations in the field data (see Fig. A5).

The TTTq DEL from measurements at tower top is, on average, overestimated by the simulations. Large variations in the loading levels, however, can be seen for positive yaw offsets and higher wind speeds both in the field data and in the predictions (see Fig. A6). Notwithstanding this discrepancy (up to $17 \%$ relative error), the increase in torque with increasing yaw misalignment is confirmed by both simulationand field-derived data.

At the tower base, FAST predicts that the resultant TBBM DELs (calculated through load roses) have a maximum between 0 and $-12.5^{\circ}$ yaw offsets. Measurements from the field, however, lead to a significant increase of the DELs with negative yaw offset $\left(-25^{\circ}\right)$ and a somewhat lesser increase for positive yaw offsets except at the higher wind speeds. The field-derived data at $-25^{\circ}$ still appear to be outliers. The largest relative error in the calculated DEL was $50 \%$ under aligned conditions and $14 \mathrm{~m} \mathrm{~s}^{-1}$.

\subsection{Extreme loads}

To complete this gallery of results, we offer a comparison between the FAST-predicted extreme loads and those measured in the field. Note that these loads are not necessarily 

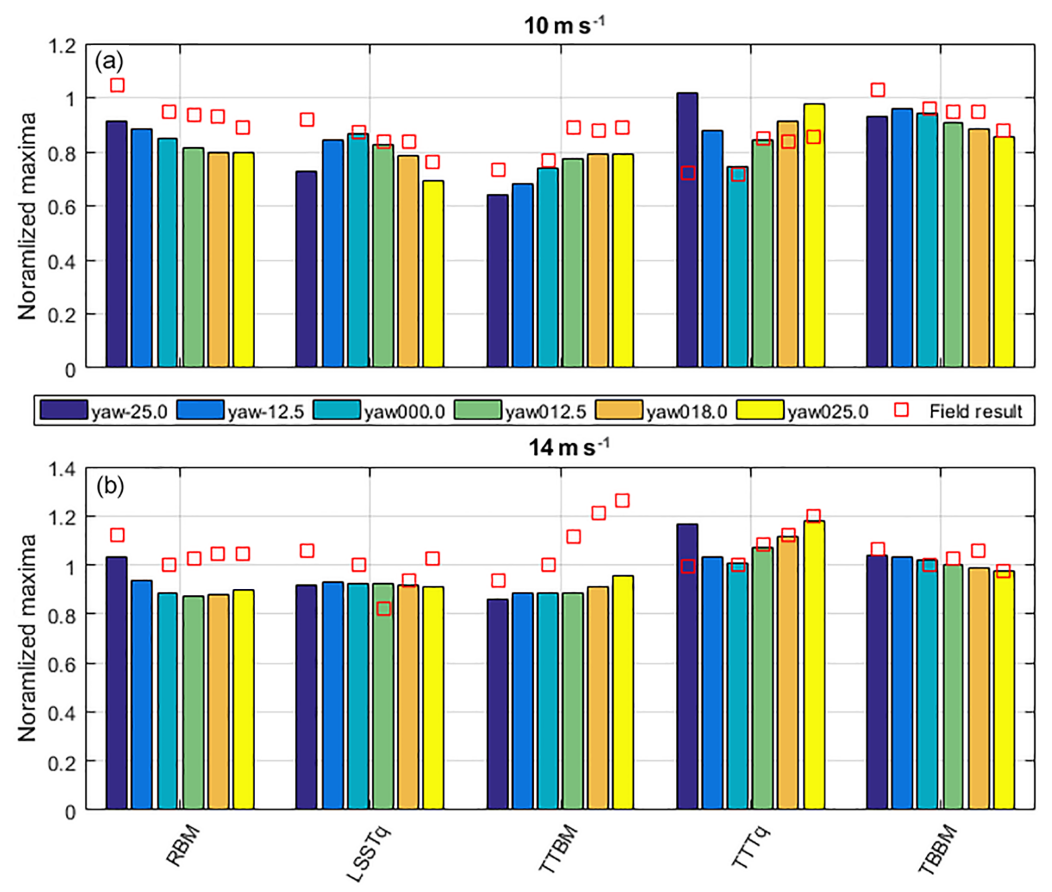

Figure 13. Comparison between predicted (FAST, bars) and measured (field, square symbols) mean extreme loads as a function of yaw offsets for mean $U_{\text {hub }}=10 \mathrm{~m} \mathrm{~s}^{-1}$ (a) and $14 \mathrm{~m} \mathrm{~s}^{-1}$ (b). The loads are normalized by their respective mean measured values at $0^{\circ}$ yaw offset and $U_{\text {hub }}=14 \mathrm{~m} \mathrm{~s}^{-1}$.

the highest loads the various components would encounter throughout the turbine lifetime, but they give an idea of what to expect in a relative sense when the turbine is operated under misaligned conditions.

Similarly to what was done for the DELs, a summary graph for the mean load values is given in Fig. 13 for all the main channels of interest. The extreme loads were normalized by the mean recorded in the field at $14 \mathrm{~m} \mathrm{~s}^{-1}$ wind speed and $0^{\circ}$ yaw. Detailed trends and graphs for individual load channels are given in Dana et al. (2017).

Except for the tower-top resultant moment (which yielded a maximum relative error of $40 \%$ at $14 \mathrm{~m} \mathrm{~s}^{-1}$ wind speed), good agreement exists between predicted and measured extreme loads.

For the RBM, the maximum values are seen at $14 \mathrm{~m} \mathrm{~s}^{-1}$, where the mean values of the load peaks increase with nonzero yaw offsets, and the largest values are predicted and measured at $\gamma=-25^{\circ}$. Note that the FAST underprediction of the loads is likely caused by the remaining difference in turbulence values between simulations and field data.

The LSSTq also shows the largest loads around rated wind speed, where both FAST and field data show limited to no effect of yaw offset.

At tower top, a distinctive increase of peak TTBM with increasing yaw offset can be observed in Fig. 13, and this trend persists in both the model and measured data. For the TTTq extremes, FAST predicts a minimum under aligned conditions, but field data show even smaller values at $\gamma=-25^{\circ}$.
Very good agreement exists for positive yaw offsets, however.

Finally, calculated TBBM extremes (highest values at $14 \mathrm{~m} \mathrm{~s}^{-1}$ wind speed) are seen to slightly decrease with increasing yaw offset. Whereas this trend is confirmed by the measurements at $10 \mathrm{~m} \mathrm{~s}^{-1}$ wind speed, at $14 \mathrm{~m} \mathrm{~s}^{-1}$ the field data are noisier and show less influence of the yaw misalignment.

\section{Conclusions}

In this paper, we presented an analytical treatment, numerical investigations, and field data analyses to explore the effects of yaw-offset operation on the loads of a commercial-grade wind turbine.

The results of models of increasing complexity were presented for the rotor aerodynamics under different yaw misalignments to shed some light on the physics and load predictions. It was shown that, under the presence of vertical wind shear, asymmetric conditions exist in the azimuthal distributions of the angle of attack and relative air velocity even under aligned inflow, which complicates the intuitive picture of the turbine response and might have further consequences on wake dynamics and control strategies. When turning the attention to the $c_{1}$ distributions, rapid changes in values obtained under both steady and unsteady aerodynamics at the inboard sections of the blades were noted for wind speeds in power region 2.5 , which are likely the responsible factors for 
the standard deviations noted in the blade-root FBMs. These effects were mostly attributed to the crossing of the stall region of the airfoil polars, which was seen as rather abrupt. At lower wind speeds (power region 2), these effects were partially eliminated by assuming rigid blades, thus demonstrating that, besides aerodynamics, the stiffness characteristics of the turbine play a significant role in the calculated DELs. A simple analytical model for the standard deviation of the out-of-plane component of the blade lift was presented and shown to be a valid proxy for DELs only under quasi-steadystate and rigid-rotor conditions. In fact, it was shown how stiffness characteristics and aerodynamics specific to the actual wind turbine model have a dominant role in the determination of the load statistics and therefore the fatigue response.

Furthermore, by considering more realistic turbulent wind fields, via both simulations and field data records, it was shown that the turbulence intensity distribution for the various mean wind-speed bins can significantly mask the effects caused by skewed inflow, and care must be given to the actual site conditions to assess their effects on the loads. Only dedicated aero-servo-elastic simulations can capture the actual trends in loading as a function of yaw offset, and only if the proper turbulent inflow conditions are matched.

Aeroelastic simulations can predict well the blade-root FBM and EBM DELs, and it was shown how these, on average, exhibit different trends with yaw offsets. Whereas the FBM increased with nonzero misalignment, the EBM decreased with increasing yaw offsets. To assess the actual impact of misaligned operation on blade-root fatigue, the combined bending, or resultant moment, was analyzed via load roses. On average, the blade-root bending moment DEL decreased for positive yaw offsets and increased for negative offsets. Fairly large variations can be attributed to different turbulence seeds and data records, making generalization more difficult.

More complex trends were observed in shaft torque and tower-top bending moment DELs. For example, trend reversals in shaft torque from the simulation-derived data were noted when the wind speed increased toward power region 3 . The initial DEL reduction with nonzero yaw offset seen for wind speeds below $10 \mathrm{~m} \mathrm{~s}^{-1}$ is replaced by a slight increase at higher wind speeds. Some of these effects are a direct consequence of the controller dynamics and therefore difficult to generalize to multiple turbine models. The resultant moment DEL at tower top increased sharply with nonzero yaw offsets in the field-derived data, whereas the FAST predictions showed little or no influence of yaw misalignment.

Tower-top torque - which, together with the tower-top bending, can be responsible for the reliability of the yaw drive components - generally showed an increase in loading with positive misalignments, exhibiting good agreement between predictions and field measurements. To reiterate, however, standard deviations were fairly large and grossly depen- dent on turbulence characteristics, which tended to override the effects of yaw misalignment.

For the tower-base bending moment, the mean DELs derived from aeroelastic simulation decrease with positive yaw offsets, whereas measurement-derived data generally indicate a slight increase. Standard deviations of the predicted DELs are, however, quite large.

Extreme loads were also compared between fieldmeasured and predicted values under operational conditions and skewed inflow. The results of the analysis showed that, for the resultant moment at the blade root, an increase can occur under misaligned conditions. The shaft torque extreme load does not significantly change with nonzero yaw offsets. Tower-top bending moments increase with increasing yaw offset, whereas tower-top torque peaks increase with nonzero misalignment. At the tower base, the resultant bending moment tends to decrease slightly with increasing yaw error.

The current dichotomy between the desire of wind power plant production optimization and the conservatism toward reliability due to load increase concerns is corroborated by the results presented in this research. In contrast to what was reported in previous studies, the fatigue load components do not necessarily decrease with increasing yaw offsets, and we offered justifications as to why that might be happening. Whereas blade-root loads can be said to decrease, large standard deviations of the mean results also point to caution and to the need to carefully assess the site conditions. Even more attention should be paid to the yaw drive system, given the measured increase in tower-top extreme loads. The turbine controller, however, can significantly impact these results.

Future work should include an extension of the analysis to other types of turbines with different aerodynamics and elastic characteristics to further assess their impact on the changes in both ultimate and fatigue loads. These additional findings will support the development of future standards for the design and certification of wind turbines that operate under programmatic yaw misalignments for increased plant performance. For example, techniques to calculate acceptable ranges for yaw offsets as a function of operating wind speeds could be provided to minimize impact on the structural design. It can also be expected that site-specific loads, obtained by using the actual site conditions and plant layout, will need to be verified against the design loads and perhaps even component test loads, during the site suitability assessment and for project certification.

Data availability. Data are available upon request. 


\section{Appendix A: Fatigue load comparisons}

Figures in Appendix A portray comparisons between predicted (FAST) and measured (field) DELs as a function of yaw offsets for mean hub-height wind speeds of 10 and $14 \mathrm{~m} \mathrm{~s}^{-1}$. DELs are normalized by the mean measured value at $0^{\circ}$ yaw offset. The error bars represent \pm 1 standard deviations.
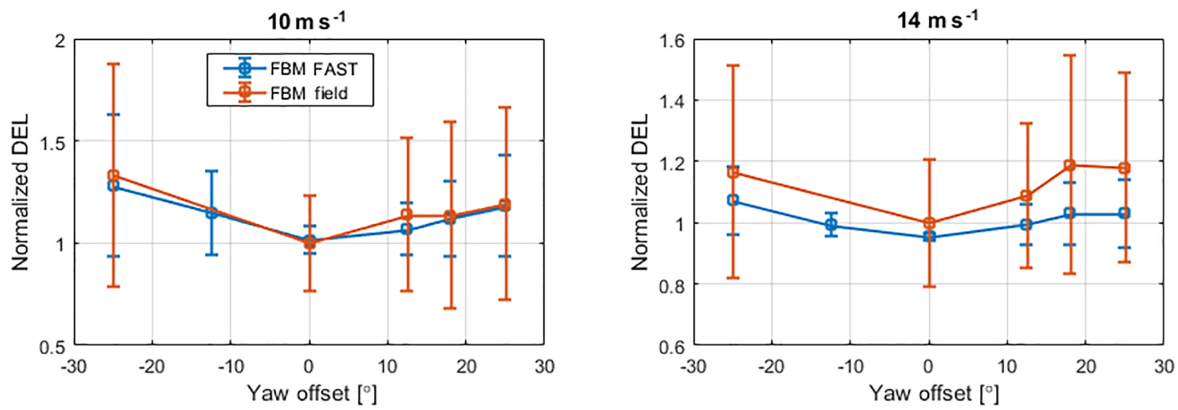

Figure A1. Comparison between predicted (FAST) and measured (field) FBM DELs.
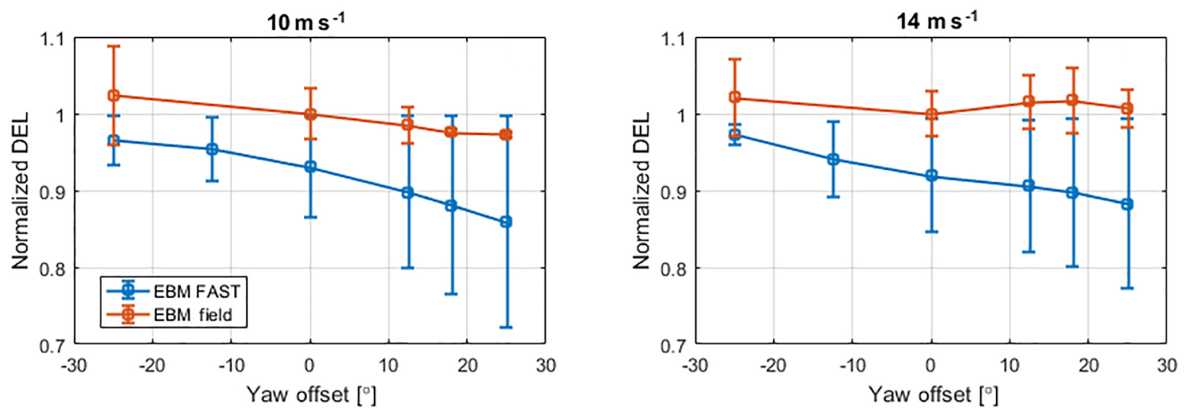

Figure A2. Comparison between predicted (FAST) and measured (field) EBM DELs.
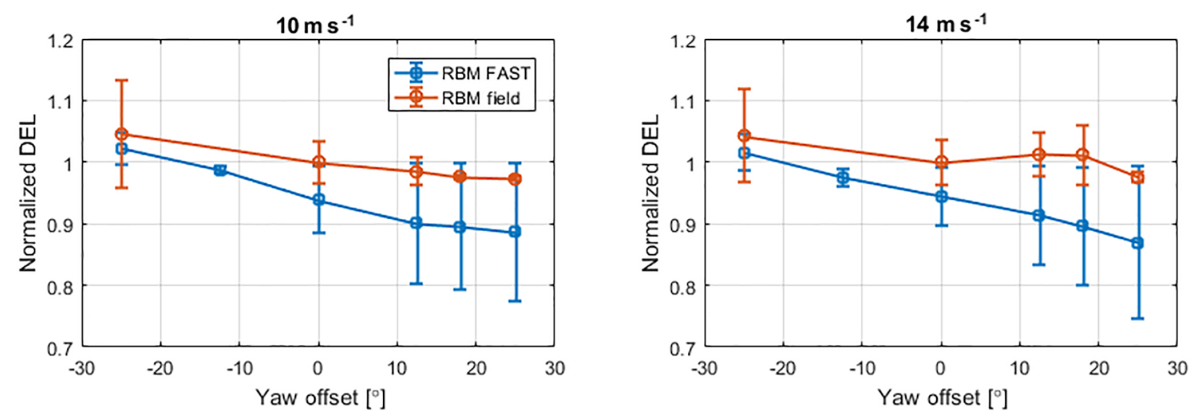

Figure A3. Comparison between predicted (FAST) and measured (field) RBM DELs. The largest component among eight load-rose sectors was selected for each yaw offset. 

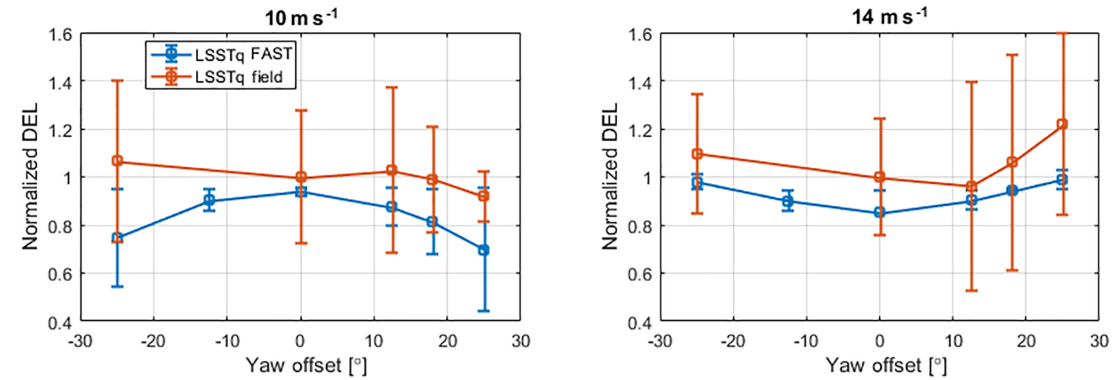

Figure A4. Comparison between predicted (FAST) and measured (field) LSSTq DELs.
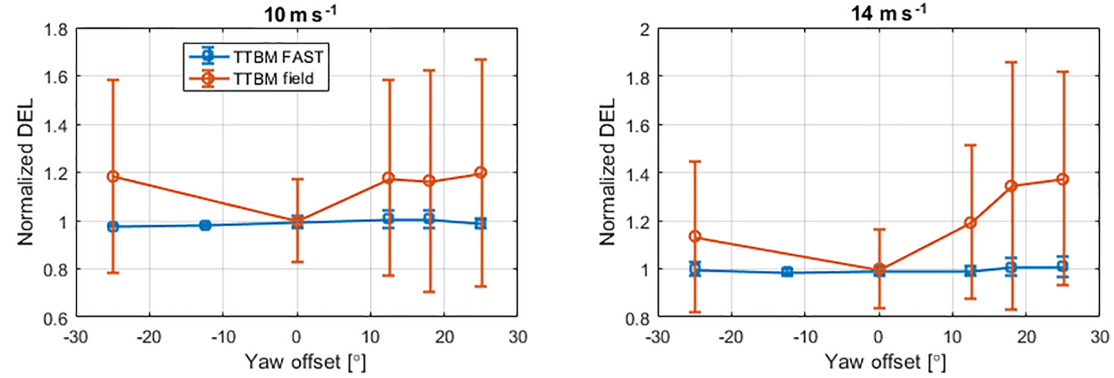

Figure A5. Comparison between predicted (FAST) and measured (field) TTBM DELs. The largest component among eight load-rose sectors was selected for each yaw offset.
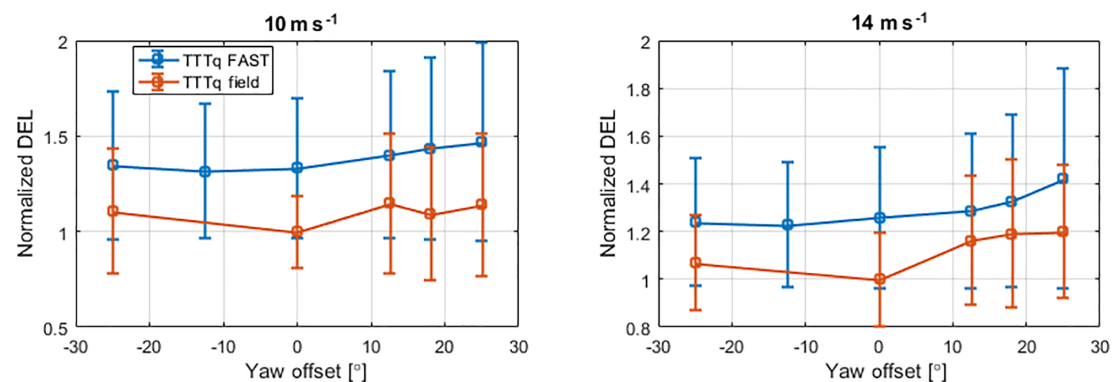

Figure A6. Comparison between predicted (FAST) and measured (field) TTTq DELs.
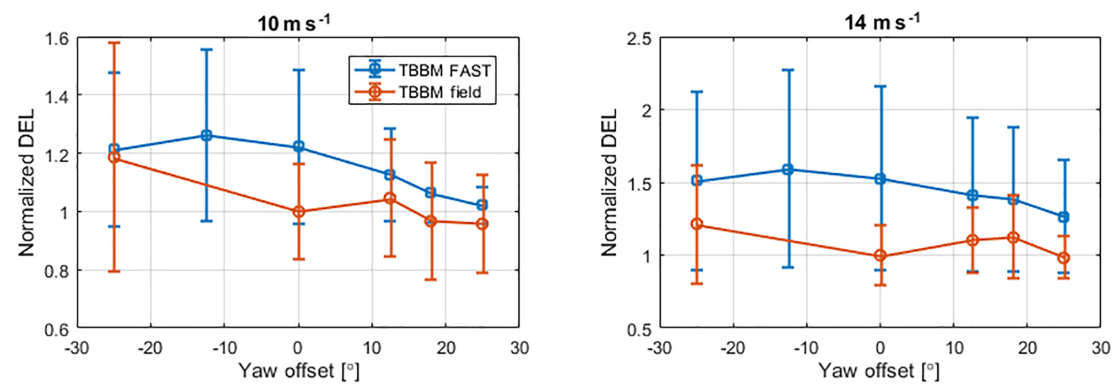

Figure A7. Comparison between predicted (FAST) and measured (field) TBBM DELs. The largest component among eight sectors was selected for each yaw offset. 
Competing interests. The authors declare that they have no conflict of interest.

Special issue statement. This article is part of the special issue "Wind Energy Science Conference 2017". It is a result of the Wind Energy Science Conference 2017, Lyngby, Copenhagen, Denmark, 26-29 June 2017.

Acknowledgements. Valuable suggestions for improving this paper came from Paul Veers and Jeroen van Dam. This work was supported by the U.S. Department of Energy under contract no. DE-AC36-08GO28308 with the National Renewable Energy Laboratory. Funding for the work was provided by the DOE Office of Energy Efficiency and Renewable Energy, Wind Energy Technologies Office.

Edited by: Gerard J. W. van Bussel

Reviewed by: Dominique Philipp Held and one anonymous referee

\section{References}

Annoni, J., Gebraad, P., Scholbrock, A., Fleming, P., and Van Wingerden, J.: Analysis of axial-induction-based wind plant control using an engineering and a high-order wind plant model, Wind Energy, 19, 1135-1150, 2016.

Bakhshi, R. and Sandborn, P.: The Effects of Yaw Error on Reliability of Wind Turbine Blades, in: PowerEnergy2016, ASME, Charlotte, NC, 2016.

Boorsma, K.: Power and loads for wind turbines in yawed conditions - Analysis of field measurements and aerodynamic predictions, Tech. Rep. ECN-E-12-047, Energy Research Center of the Netherlands, the Netherlands, 2012.

Dahlberg, J. and Medici, D.: Potential improvement of wind turbine array efficiency by active wake control, in: Proceedings of European Wind Energy Conference, 65-84, Madrid, Spain, 2003.

Damiani, R., Hayman, G., and Jonkman, J.: Development and Validation of a New Unsteady Airfoil Aerodynamics Model Within AeroDyn, in: 34th Wind Energy Symposium-SciTech 2016, AIAA, San Diego, CA, 2016.

Dana, S., Damiani, R., and Dykes, K.: Field Measurement Campaign of the GE 1.5-SLE under Yawed Inflow, Technical Report NREL/TP-5000-XXXXX, NREL, Golden, CO, in preparation, 2017.

Downing, S. D. and Socie, D. F.: Simple rainflow counting algorithms, Int. J. Fatigue, 4, 31-40, 1982.

Ekelund, T.: Yaw control for reduction of structural dynamic loads in wind turbines, J. Wind Eng. Ind. Aerod., 85, 241-262, 2000.

Fleming, P., Scholbrock, A., Jehu, A., Davoust, S., Osler, E., Wright, A., and Clifton, A.: Field-test results using a nacelle-mounted lidar for improving wind turbine power capture by reducing yaw misalignment, Journal of Physics: Conference Series, 524, 012002, https://doi.org/10.1088/17426596/524/1/012002, 2014.

Fleming, P., Gebraad, P. M. O., Lee, S., van Wingerden, J., Johnson, K., Churchfield, M., Michalakes, J., Spalart, P., and Moriarty, P.:
Simulation comparison of wake mitigation control strategies for a two-turbine case, Wind Energy, 18, 2135-2143, 2015.

Frishman, F.: A Modern Course on Statistical Distributions in Scientific Work: Volume 1 - Models and Structures Proceedings of the NATO Advanced Study Institute held at the University of Calgary, Calgary, Alberta, Canada 29 July10 August 1974, chap. On the Arithmetic Means and Variances of Products and Ratios of Random Variables, 401-406, Springer Netherlands, Dordrecht, https://doi.org/10.1007/97894-010-1842-5_32, 1975.

Gebraad, P. M. O., Teeuwisse, F. W., van Wingerden, J. W., Fleming, P. A., Ruben, S. D., Marden, J. R., and Pao, L. Y.: Wind plant power optimization through yaw control using a parametric model for wake effects - a CFD simulation study, Wind Energy, 19, 95-114, 2016.

Gebraad, P., Thomas, J. J., Ning, A., Fleming, P., and Dykes, K.: Maximization of the annual energy production of wind power plants by optimization of layout and yaw-based wake control, Wind Energy, 20, 97-107, 2017.

Hand, M. M., Simms, D. A., Fingersh, L. J., Jager, D. W., Cotrell, J. R., Schreck, S., and Larwood, S. M.: Unsteady Aerodynamics Experiment Phase VI: Wind Tunnel Test Configurations and Available Data Campaigns, Tech. Rep. NREL/TP-500-29955, National Renewable Energy Laboratory, Golden, CO, contract No. DE-AC36-99-GO10337, 2001.

Huang, L.: Fatigue Load Calculations for ROMO Wind to Assess Sensitivity to Changes in 10-min Mean Yaw Error, Tech. rep., GL Garrad Hassan, Bristol, UK, 2012.

IEC: 61400-1. Wind turbines - Part 1: Design requirements, Geneva, Switzerland, 2005.

IEC: 61400-13:2015 Wind turbines - Part 13: Measurement of mechanical loads, Geneva, Switzerland, 2015.

IEC: 61400-12-1:2017 Wind energy generation systems - Part 12-1: Power performance measurements of electricity producing wind turbines, Geneva, Switzerland, 2017.

Jiménez, A., Crespo, A., and Migoya, E.: Application of a LES technique to characterize the wake deflection of a wind turbine in yaw, Wind Energy, 13, 559-572, 2010.

Jonkman, J. M., Hayman, G. J., Jonkman, B. J., and Damiani, R. R.: AeroDyn v15 User's Guide and Theory Manual, Tech. rep., National Renewable Energy Laboratory, Golden, CO, 2015.

Kragh, K. A. and Hansen, M. H.: Load Alleviation of wind turbines by yaw misalignment, Wind Energy, 17, 971-982, https://doi.org/10.1002/we.1612, 2014.

Kragh, K. A. and Hansen, M. H.: Potential of power gain with improved yaw alignment, Wind Energy, 18, 979-989, https://doi.org/10.1002/we.1612, 2015.

Mendoza, I., Hur, J., Thao, S., and Curtis, A.: Power Performance Test Report for the U.S. Department of Energy 1.5-Megawatt Wind Turbine, Tech. Rep. TP-5000-63684, NREL, Golden, CO, 2015.

Mortimer, A.: New ideas for improving wind-farm outputs, Windpower Engineering \& Development, 46-50, 2016.

Ning, S. A., Hayman, G., Damiani, R., and Jonkman, J.: Development and Validation of a New Blade Element Momentum Skewed-Wake Model within AeroDyn, in: AIAA SciTech 2015 - 33rd Wind Energy Symposium, AIAA, Kissimmee, FL, https://doi.org/10.2514/6.2015-0215, 2015. 
Schepers, J.: Annexlyse: Validation of Yaw Model, on Basis of Detailed Aerodynamic Measurements on Wind Turine Blades, Tech. Rep. ECN-C-04-097, Energy Research Center of the Netherlands, the Netherlands, 2004.

Schepers, J.: Engineering models in wind energy aerodynamics: Development, implementation and analysis using dedicated aerodynamic measurements, PhD thesis, TU Delft, the Netherlands, https://doi.org/10.4233/uuid:92123c07-cc12-4945973f-103bd744ec87, 2012.

Schepers, J., Boorsma, K., Cho, T., Gomez-Iradi, S., Schaffarczyk, P., Jeromin, A., Shen, W. Z., Lutz, T., Meister, K., Stoevesandt, B., Schreck, S., Micallef, D., Pereira, R., Sant, T., Madsen, H. A., and Sørensen, N.: Final report of IEA Task 29, Mexnext (Phase 1) - Analysis of Mexico wind tunnel measurements, Tech. Rep. ECN-E-12-004, Energy Center of the Netherlands, 2011.

Schreck, S. J. and Schepers, J.: Unconventional Rotor Power Response to Yaw Error Variations, Journal of Physics: Conference Series, 555, 012001, https://doi.org/10.1088/17426596/555/1/012001, 2014.
Thomsen, K. and Sørensen, P.: Fatigue loads for wind turbines operating in wakes, J. Wind Eng. Ind. Aerod., 80, 121-136, 1999.

Vollmer, L., Steinfeld, G., Heinemann, D., and Kühn, M.: Estimating the wake deflection downstream of a wind turbine in different atmospheric stabilities: an LES study, Wind Energ. Sci., 1, 129141, https://doi.org/10.5194/wes-1-129-2016, 2016.

Wagenaar, J., Machielse, L., and Schepers, J.: Controlling wind in ECN's scaled wind farm, in: Proceedings of the EWEA Annual Meeting, Copenhagen, Denmark, 2012.

Zalkind, D. S. and Pao, L. Y.: The Fatigue Loading Effects of Yaw Control for Wind Plants, in: 2016 American Control Conference, 537-542, ACC, Boston Marriot Copley Place, Boston, MA, 2016. 